\title{
Acculturation, HIV-Related Stigma, Stress, and Patient-Healthcare Provider Relationships Among HIV-Infected Asian Americans: A Path Analysis
}

\author{
Feifei Huang ${ }^{1,2} \cdot$ Wei-Ti Chen ${ }^{2}$ (1) $\cdot$ Cheng-Shi Shiu ${ }^{2,3} \cdot$ Wenxiu Sun ${ }^{2,4} \cdot$ Lance Toma $^{5} \cdot$ Binh Vinh Luu $^{6} \cdot$ Judy Ah-Yune $^{6}$
}

Published online: 13 August 2020

○) Springer Science+Business Media, LLC, part of Springer Nature 2020

\begin{abstract}
Acculturation may limit HIV-positive Asian Americans' active interactions with patient-healthcare providers (HCP) and utilization of HIV healthcare services; however, the specific mediation effect of acculturation still unknown. A bias-corrected factor score path analysis was performed to examine the proposed model of relations among acculturation, stigma, stress, and patient-HCP relationships. A convenience sample of 69 HIV-positive Asian Americans in San Francisco, Los Angeles, and New York City were recruited and collect data were collected on demographics, HIV-related stigma, stress, and patient-HCP relationships. HIV stigma and stress had a direct, negative effect on patient-HCP relationships. Acculturation had a positive total effect on patient-HCP relationships, and was mediated by HIV stigma and stress. A acculturation also had a direct impact on stigma and stress. Acculturation, HIV-related stigma, and stress are key elements to achieving good patient-HCP relationships, and provide insights on the design of culturally sensitive interventions to improve patient-HCP relationships.
\end{abstract}

Keywords HIV $\cdot$ Acculturation $\cdot$ Stigma $\cdot$ Patient-healthcare provider relationships $\cdot$ Asian americans

\section{Background}

Asian Americans are one of the fastest growing minority groups in the United States [1], and they have been disproportionately affected by the HIV epidemic [2]. According to the most recent U.S. census, Asian Americans represent $5.4 \%$ of the total U.S. population at close to 17.2 million [1]. Between 2010 and 2016, the Asian population in the

Wei-Ti Chen

wchen@sonnet.ucla.edu

1 School of Nursing, Fujian Medical University, Fuzhou, China

2 School of Nursing, University of California Los Angeles, Los Angeles, CA 90095, USA

3 Department of Social Work, National Taiwan University, Taipei, Taiwan

4 Shanghai Public Health Clinical Center, Fudan University, Shanghai, China

5 San Francisco Community Health Center, San Francisco, CA, USA

6 Chinese-American Planning Council, Inc, New York, NY, USA
U.S. grew approximately $17 \%$, yet the rate of HIV diagnosis among this group increased by nearly $70 \%$ [2]. In addition, when diagnosed, Asian Americans are typically already at a late stage of the pre-AIDS infection and usually go on to develop full-blown AIDS within a short time [3]. Similar to other people living with HIV/AIDS (PLWHA), HIV-infected Asian Americans may also suffer from HIV-related stigma and stress, which in turn influences their patient-healthcare provider (HCP) relationships [4, 5].

The patient-HCP relationship refers to information sharing between patients and providers, shared responsibilities in decision-making processes, and support of patient choices [6]. In Asian culture, Asian PLWHA often seek physical and emotional support from HCP when support from friends or family is lacking due to HIV-related shame and stigma [7]. This is unfortunate, since HCP have the knowledge and expertise to treat the disease and instruct patients and family members in how to take care of HIV-related physical and mental symptoms [5]. Studies have identified that many PLWHA have unfavorable patient-HCP relationships $[8,9]$. As a result, communication suffers, which leads in turn to poorer quality of care, lower compliance with treatment regimens, decreased the self-efficacy of taking medication [8], and worse patient satisfaction and quality of life 
[9]. However, specific research on how to improve the relationships between Asian American PLWHA and HCP to enhance healthcare engagement is limited $[4,10]$.

In the United States, the highly diverse ethnic Asian population includes Chinese, Filipinos, Indians, Vietnamese, Koreans, Taiwanese, and Japanese, among others [11]. Asian PLWHA may not only suffer from HIV-related stress and stigma but also have additional stress from acculturation to U.S. customs [12]. Acculturation is defined as a process of change that occurs when individuals come from different cultures and interact with the host regions, which is usually developed by following migration, political conquest, or forced relocation [12]. HIV-infected Asian Americans also face myriad difficulties related to acculturation, such as acculturation stress (e.g. poor English language ability), health access difficulties, stigma, serostatus disclosure issues, limited access to medications, and continuation of family obligations [3,11-13]. Studies have shown that the level of acculturation is associated with immigrants' stress [12], stigma, and health-related quality of life [14, 15]. Also, for those Asian PLWHA who have a low or moderate acculturation level to U.S. culture, at least one study has shown that stress is significantly mediated by depression symptoms [12]. Thus, the level of acculturation can limit Asian Americans' active interactions with HCP and use of HIV healthcare services [16].

Obviously, it is understood that acculturation (i.e. the learned behaviors, beliefs, and attitudes toward the host country) play a crucial role in immigrants' health [17]. Several acculturation models describe the acculturation process and its implications for immigrants' well-being. Berry's acculturation stress theory addresses the influence of both cultural norms and stress, which is useful in understanding the relationship between acculturation and health-related behaviors [18]. PLWHA who are immigrants have to face stressful situations such as discrimination and language barriers in the process of adapting to the receiving culture, which makes them highly vulnerable to maladaptive behaviors. Furthermore, Choi's cultural marginality model states that people emigrating to the United States from non-Western cultures may have difficulty adhering to the U.S. medical system, which consequently impacts their well-being and their relationships with HCP [19]. Based on this, we conclude that relationships between HCP and immigrants can be influenced by their level of acculturation; however, there is still a lack of understanding as to how the acculturation leverages HIV-stigma, stress, and patient-HCP interactions among Asian PLWHA [4].

To address this gap, we used path analysis to understand the impact of acculturation on the relationships among stigma, stress and HCP for Asian immigrants. This will provide insight into potential mechanisms for strengthening and promoting positive interactions between Asian Americans PLWHA and HCP. Based on the acculturation framework and other reports, we proposed the pathway of acculturation to patient-HCP relationships through selected psychological factors (HIV-related stigma and stress) depicted in Fig. 1 [4, $12,20]$. We hypothesized that acculturation can impact all the components of the stigma $\rightarrow$ stress $\rightarrow$ HCP pathway and found direct evidence for two (acculturation $\rightarrow$ stigma and acculturation $\rightarrow$ stress). Acculturation can also indirectly impact HCP through the stigma and stress pathways.

Fig. 1 Hypothesized pathway

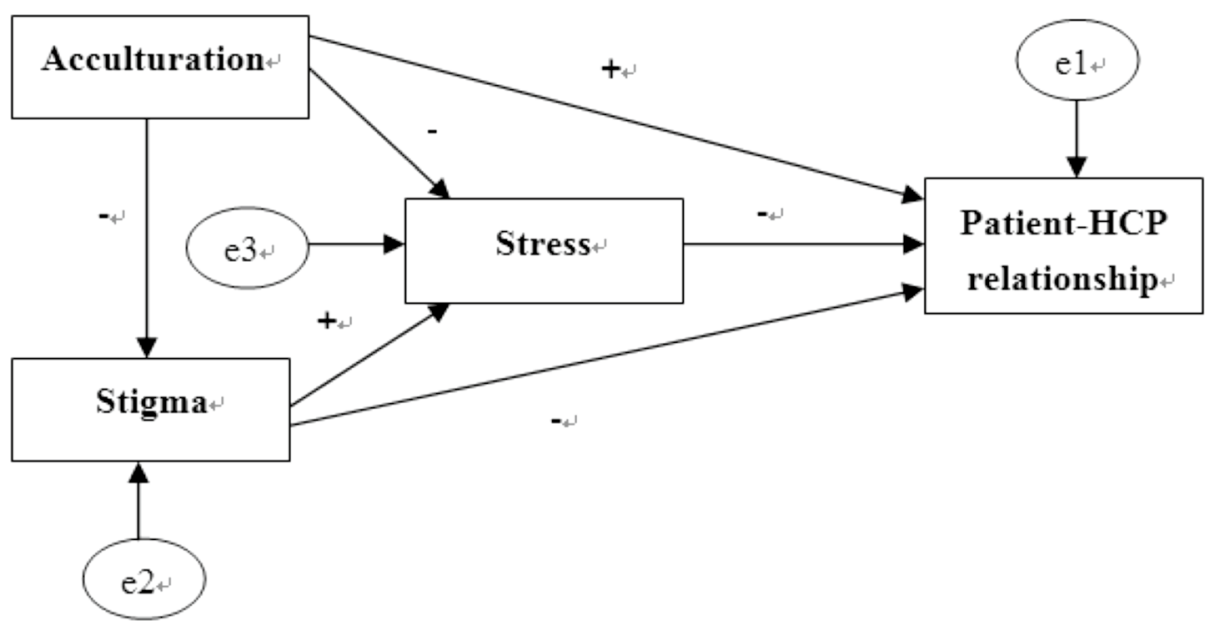

Note: Observed variables are shown within rectangles

Directional relationships are indicated with +/- 


\section{Methods}

\section{Sample, Settings, and Procedures}

The study had two phases: (1) From January to June 2013, a convenience sample of 50 HIV-positive Asian Americans was recruited in two cities (San Francisco and New York City). Participants were recruited from the Asian \& Pacific Islander Wellness Center (A\&PI Wellness Center) in San Francisco, the Chinese-American Planning Council, Inc. (CPC), and the Asian \& Pacific Islander Coalition on HIV/ AIDS Community Health Center (APICHA Community Health Center) in New York City. (2) From September 2017 to January 2020, we recruited an additional 19 Asian American PLWHA from the CPC and Asian Pacific American AIDS Intervention Team (APAIT) in Los Angeles. A total of 69 Asian Americans participated in this study analysis.

The relevant institutional ethical review boards approved this study. The study inclusion criteria were as follows: (a) self-identified as Asian or Pacific Islanders, (b) confirmed HIV serostatus, (c) willing to participate in the survey, and (d) at least 18 years old. After securing consent from the participants, cross-sectional audio computer-assisted selfinterviews (ACASIs) or Research Electronic Data Captures (REDCaps) were conducted with each participant. All participants received a small reimbursement for their participation.

\section{Measures}

Participants completed a 60-min ACASI survey that consisted of standardized measures to assess demographics, HIV-related perceived stress, stigma, acculturation, and patient-HCP relationships. These measures have been tested in Asian populations and have shown strong reliability and validity over time [6,21-24].

\section{Demographics}

Participant age, gender, marital status, ethnicity, educational level, working status, immigrant status, years of living with HIV, HIV medication, and recent viral load were collected.

\section{Engagement with HCP scale}

This 13-item scale is designed to assess how individuals rate their interaction with their HCP (e.g. "My HIV doctor answers my questions," "My HIV doctor cares about me," and so on). Each item was measured on a 4-point scale $(1=$ always true to $4=$ never true). Items were reverse-scored and averaged to create a composite, with higher scores representing better patient-HCP relationships [6]. The overall Cronbach's alpha reliability estimate for this sample was 0.92 .

\section{Suinn-Lew Asian Self-Identity Acculturation Scale}

This 21-item scale measures six acculturation issues in Asian populations: food preferences, social friend's ethnicity, TV programs and language preferences, music selection, length of residency in the United States, and age at arrival $[21,24]$. Score ranges are from 1.00 (low acculturation) to 5.00 (high acculturation). For this scale, the overall Cronbach's alpha reliability was 0.96 .

\section{HIV Berger Stigma Scale}

This 40-item scale measures how stigma operates within individuals and how it can affect them. It covers personal stigma, disclosure concerns, negative self-image, and concerns with public attitudes toward people with HIV. Each stigma item can be rated using a 4-point Likert-type scale (strongly disagree, disagree, agree, or strongly agree), with higher values indicating greater stigma $[6,22]$. For this scale, the overall Cronbach's alpha reliability was 0.95 .

\section{Perceived Stress Scale in HIV (PSSHIV)}

This 44-item scale measures perceived stress levels elevate in eight domains: social/psychological problems, sexual relationships, functional problems, social acceptance/rejection issues, work-related issues, family/offspring issues, accessibility to treatment, and treatment outcomes. Items are rated on a 5-point Likert scale, from " $1=$ absolutely not stressful" to "5-extremely stressful" [23]. For this scale, the overall Cronbach's alpha reliability was 0.97 .

\section{Analysis}

Data analyses were conducted using SPSS 24.0 (IBM, Chicago, IL) and the Lavaan package in R. In the present study, the data met the assumptions of normality (one sample Kolmogrov-Smirnov test did not show statistical significance). The continuous variables were expressed as means and standard deviations (SD). Categorical variables were expressed as proportions or percentages. Pearson's correlation analysis was conducted to examine the relationships among acculturation, HIV-related stress, stigma, and patientHCP relationships. Missing data were replaced using full information maximum likelihood; $p<0.05$ was considered significant.

Due to the sample size in this study, we used bias-corrected factor score path analysis, which has been shown to produce unbiased and efficient estimates in small to 
moderate sample settings [25]. We tested the pathways in three steps. First, we estimated the variance-covariance matrix of the factor scores along with acculturation, HIVrelated stress, stigma, and patient-HCP relationships. Second, we corrected the covariance matrix to obtain an estimate of the true covariances. Finally, we performed path analysis to test the hypothesized pathway (Fig. 1) [26] and obtained unbiased estimates of the true structural path coefficients. The following model-fit indices were used: normed chi-square ( $\left.\chi^{2} / \mathrm{df}, 1.0 \pm 3.0, p>0.05\right)$, root mean squared error of approximation (RMSEA $<0.08$ ), comparative fit index (CFI, $>0.9$ ), and Tucker-Lewis index (TLI, $>0.9$ ). To achieve a more parsimonious model, we further removed parameters that did not significantly differ from zero. We also adjusted the estimates by controlling for significant demographic variables: marital status, ethnicity, educational level, working status, immigrant status, and HIV medication (see Table 1). Finally, to investigate the relationships between acculturation and patient-HCP relationships through different pathways, we applied the bootstrap method (repeated 1,000 times) to obtain more stable and valid standard errors of the estimates of the direct and indirect effects
Table 1 Socio-demographic characteristics of participants $(n=69)$

\begin{tabular}{|c|c|c|c|c|c|}
\hline \multirow[t]{2}{*}{ Variables } & \multirow[t]{2}{*}{$N(\%)$} & \multicolumn{4}{|l|}{$t / F$ value } \\
\hline & & Acculturation & HIV-related stress & Stigma & $\begin{array}{l}\text { Patient-HCP } \\
\text { relationships }\end{array}$ \\
\hline Gender & & 1.27 & -0.72 & -1.86 & 0.68 \\
\hline Male & $54(78.30 \%)$ & & & & \\
\hline Ethnicity & & $14.99^{\mathrm{a}}$ & $4.15^{\mathrm{a}}$ & $3.5^{\mathrm{a}}$ & 1.26 \\
\hline Chinese & $35(50.70 \%)$ & & & & \\
\hline Filipino & $11(15.90 \%)$ & & & & \\
\hline Japanese & $3(4.30 \%)$ & & & & \\
\hline Malaysian & $5(7.20 \%)$ & & & & \\
\hline Indonesian & $1(1.40 \%)$ & & & & \\
\hline Vietnamese & $7(10.10 \%)$ & & & & \\
\hline Other & $7(10.10 \%)$ & & & & \\
\hline Are you an immigrant & & $4.60^{\mathrm{a}}$ & -1.73 & 0.03 & $4.26^{\mathrm{a}}$ \\
\hline Yes & $63(91.30 \%)$ & & & & \\
\hline Education & & $10.74^{\mathrm{a}}$ & 0.52 & $2.6^{\mathrm{a}}$ & 0.74 \\
\hline 11 th grade or less & $33(47.80 \%)$ & & & & \\
\hline High school or GED & $20(29.00 \%)$ & & & & \\
\hline 2 years of college/AA degree & $10(14.50 \%)$ & & & & \\
\hline College & $5(7.20 \%)$ & & & & \\
\hline Doctorate & $1(1.40 \%)$ & & & & \\
\hline Currently working status & & 3.53 & 2.89 & 0.11 & 1.08 \\
\hline No & $49(71.00 \%)$ & & & & \\
\hline Part time & $10(14.50 \%)$ & & & & \\
\hline Full time & $10(14.50 \%)$ & & & & \\
\hline Marital status* & & $11.03^{\mathrm{a}}$ & 0.33 & 1.49 & 0.16 \\
\hline Married & $22(32.80 \%)$ & & & & \\
\hline Divorced & $7(10.40 \%)$ & & & & \\
\hline Single & $33(49.30 \%)$ & & & & \\
\hline Cohabited & $3(4.50 \%)$ & & & & \\
\hline Widowed & $2(3.00 \%)$ & & & & \\
\hline Currently Using ART & & -0.43 & $2.39^{\mathrm{a}}$ & 0.70 & -0.74 \\
\hline Yes & $66(95.70 \%)$ & & & & \\
\hline Recent viral load* & & 1.45 & 2.87 & 1.60 & 2.54 \\
\hline Undetectable & $50(73.50 \%)$ & & & & \\
\hline Detectable & $7(10.30 \%)$ & & & & \\
\hline Don't know & $11(16.20 \%)$ & & & & \\
\hline
\end{tabular}

*Missing data

${ }^{\mathrm{a}} p<0.05$ 
of these factors. Standardized regression coefficient $(\beta)$ and $p$ values for $\beta$ of direct, indirect, and total effects were identified and reported by path analysis. Also, to compare the modified pathway with the initial pathway, we applied the Akaike information criterion (AIC) [27].

\section{Results}

\section{Descriptive and Bivariate Analysis}

Demographic characteristics are presented in Table 1. Among the total sample of 69 HIV-infected Asian Americans, $78.30 \%$ were male $(N=54)$, with a mean age of 51 years $(S D=10.50$, range $=31-72)$, and average years of living with HIV of 33 years $(S D=14.54)$. Results of the bivariate analyses are summarized in Table 2. The results suggest that acculturation, HIV-related stress, stigma, and patient-HCP relationships are significantly correlated with each other.

Table 2 Bivariate correlation among variables

\begin{tabular}{llcl}
\hline & $\begin{array}{l}\text { Patient-HCP } \\
\text { relationship }\end{array}$ & Stress & Stigma \\
\hline Patient-HCP relationship & - & & \\
Stress & $-0.281^{*}$ & - & \\
Stigma & $-0.257^{*}$ & $0.507^{* *}$ & - \\
Acculturation & $0.537^{* *}$ & $-0.250^{*}$ & $-0.399^{* *}$ \\
\hline${ }^{*} p<0.05$ & & & \\
${ }^{* * *} p<0.01$ & & & \\
& & &
\end{tabular}

\section{Pathway Analysis}

It was shown by the fit indices that for the proposed model $\chi^{2}(18)=23.99, p=0.16$, RMSEA $=0.07(P$-Close $=0.31)$, $\mathrm{CFI}=0.96 \& \mathrm{TLI}=0.86$, suggesting that the proposed pathway fit well to the data. Most of the hypothesized relationships were significant and supported by the data; however, because the relationship between acculturation and patient-HCP relationships was insignificant $(p>0.05)$, we further suppressed this relationship to zero and tested the structural relationships of the model again. Compared to the proposed model, the model-fit indices of the final model were improved $\left(\chi^{2}(2)=1.36, p=0.00, \mathrm{RMSEA}=0.000\right.$ $(P$-Close $=0.28), \mathrm{CFI}=0.99, \& \mathrm{TLI}=0.94)$. The reduced model was further supported by a smaller AIC value (proposed: 30.56 vs. reduced: 27.36 ), indicating a better fit. The coefficients for all paths are shown in Fig. 2.

Table 3 summarizes the standardized direct, indirect, and total estimates of the reduced model's paths. According to the model, HIV stigma ( $\beta=-0.154)$ and stress $(\beta=-0.203)$ had a negative direct effect on patient-HCP relationships $(p<0.05$ for the model). Although acculturation had no significant direct effect on patient-HCP relationships, it still had a positive total effect $(\beta=0.094)$, which can be seen to be mediated by HIV stigma (indirect $\beta=-0.06$ ) and stress (indirect $\beta=-0.01$ ). Moreover, acculturation directly impacted stigma $(\beta=$

$-0.399)$ and stress $(\beta=-0.048)$.

Consistent with the hypothesis, $20.30 \%$ and $15.40 \%$ of the variance in patient-HCP relationships were negatively predicted by HIV-related stress and stigma, respectively. In addition, $39.90 \%$ of the variance in HIV-related stigma was
Fig. 2 Final standardized parameter estimates model

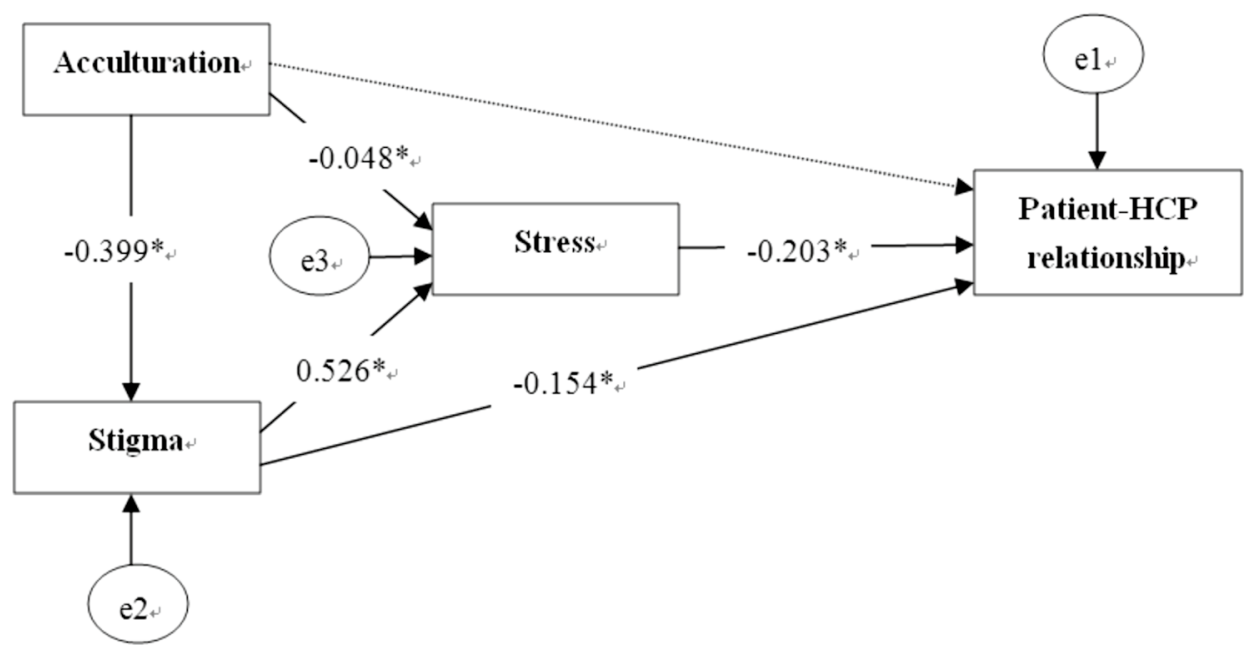

Note: ${ }^{*} p<0.05$; Controlling for marital status, ethnicity, education level, working status, immigrant status, and HIV medication; Dashed line indicates that the direct relationship between acculturation and patient-HCP relationship was nonsignificant $(p>0.05)$. 
Table 3 Effect coefficients of the final model

\begin{tabular}{lllll}
\hline Endogenous variables & Predicting variables & $\begin{array}{l}\text { Standardized } \\
\text { direct effect } \beta\end{array}$ & $\begin{array}{l}\text { Standardized } \\
\text { indirect effect } \beta\end{array}$ & $\begin{array}{l}\text { Standardized } \\
\text { total effect } \beta\end{array}$ \\
\hline Patient-HCP relationship & Stigma & $-0.154^{*}$ & $-0.107^{*}$ & $-0.261^{*}$ \\
& Stress & $-0.203^{*}$ & - & $-0.203^{*}$ \\
& Acculturation & - & $0.094^{*}$ & $0.094^{*}$ \\
Stress & Acculturation & $-0.048^{*}$ & $-0.210^{*}$ & $-0.258^{*}$ \\
& Stigma & $0.526^{*}$ & - & $0.526^{*}$ \\
Stigma & Acculturation & $-0.399^{*}$ & - & $-0.399^{*}$ \\
\hline
\end{tabular}

${ }^{*} p<0.01$ negatively predicted by acculturation; $52.60 \%$ and $4.80 \%$ of the variance in HIV-related stress were positively predicted by stigma and acculturation, respectively.

\section{Discussion}

Our data and pathways show acculturation to be an important factor in the patient-HCP relationship, which is also echoed by other reports $[4,28]$. This is also one of the initial reports to offer pathway evidence that links positive acculturation to patient-HCP relationships in Asian Americans. Coinciding with the explanations provided by Berry and Choi's models $[18,19]$, these hypotheses of the relationships among study variables is supported, except for the direct effect of acculturation and patient-HCP relationships. The present study showed that acculturation is beneficial for patient-HCP relationships to the extent that it decreases perceived stigma and stress in Asian American PLWHA. In other words, for Asian American PLWHA, focusing on enhancing acculturation is warranted, as it is a potential mechanism to reduce HIV-related stigma and stress. Consequently, acculturation can enhance patient-HCP relationships. As such, the present study's findings highlight the potential utility of targeting acculturation in interventions aimed at improving patientHCP relationships.

This study confirms the acculturation-stress-patient-HCP relationships pathway. As stipulated by the transactional theory of stress, stress is a result of individuals' appraising a potential stressor as negative and beyond his or her coping resources [29]. The Asian American PLWHAs remained attached to their original cultures while developing connections with the host country [12], and thus they face the perceived stress of acculturation just as other immigrants do. Acculturation stress can include family pressure, the potential for inadvertent disclosure to the public, and other challenges $[12,30]$. If Asian American PLWHA perceive acceptance into the dominant culture as a daunting undertaking, the process of integrating to the host country becomes a stressor [12]. On the other hand, if they perceive acceptance into the dominant culture as neutral or positive, and thus manageable, the acculturation process is less stressful and can be seen as a positive occurrence [20]. The results can lead to worse or closer patient-HCP relationships [30].

The hypothesis that the effect of acculturation on patient$\mathrm{HCP}$ relationships is mediated by HIV-related stigma was also sustained. This could be due to the internalization of HIV-related misconceptions from the country of origin, such as the belief that HIV is "dirty" and that HIV is in effect a death penalty [31]. Limited English language ability and other acculturation challenges, such as social isolation and lack of self-management, can worsen PLWHA's own internalized stigma and shame [14]. Therefore, their trust of HCP and HIV care in the host country could be jeopardized [32].

The Joint United Nations Programme on HIV/ AIDS (UNAIDS) has set a 90-90-90 target for all countries to scale up testing and treatment; thus, by $2020,90 \%$ of PLWHA will know their status; $90 \%$ of those diagnosed will be on antiretroviral therapy, and $90 \%$ of those receiving antiretroviral medications will be virally suppressed [33]. However, to reach this target, reducing stigma and discrimination are urgently needed [33]. Thus, culturally tailored interventions to reduce HIV-related stigma should be tested and implemented, not only to enhance the acculturation process, but also to better engage Asian American PLWHA in managing their own health care.

Currently, interventions aimed at improving immigrant patients' HCP relationships are lacking, and there are limited studies to support the development of such interventions [4]. Thus, the present study could be important for developing a feasible, acceptable and effective, culturally sensitive intervention to improve patient-HCP relationships. This study shows that acculturation-stress and stigma-patient HCP relationship pathways are highly influential. Therefore, when designing an intervention to enhance patient-HCP relationships, these factors should be considered, because acculturation, HIV-related stigma and stress are key to achieving therapeutic patient-HCP relationships. Due to their typically heavy workload and lack of time to devote to cultural sensitivity, HCP may draw on stereotypical assumptions when they encounter people who are not fluent in English and may be naïve about the U.S. healthcare system [8]. As a 
result, HCP are less likely to assess individual needs, such as acculturation levels, stigma and stress [8]. This can lead to care disparities in the areas of both physical and psychological health, diminishing the overall quality of care [4]. Thus, supporting providers in their efforts to create mutually beneficial patient-HCP relationships can influence the quality of care and result in better health for PLWHA [10]. By providing tailored training to HCP on measuring acculturation levels as well as psychosocial needs and barriers, HCP can enhance healthcare engagement among Asian American PLWHA $[4,10]$.

This study has several limitations. First, the proposed pathway does not explain the majority of variances in patient-HCP relationships, including variances in depression, which were not considered in this model. Besides, there might be an overfitting of the pathway due to six covariates that were adjusted for in the pathway: according to the rule of thumb, one covariate per 10 subjects, although we used bias-corrected factor score path analysis, which has been shown to produce unbiased and efficient estimates in small to moderate sample settings. Second, the convenience sampling method and a small sample size limits the generalizability of the findings; however, that must be measured against the fact that Asian American PLWHA are one of the hardest-to-reach populations, and this study is one of the pioneering studies in the field. Last, this is a cross-sectional study and causality cannot be inferred. Therefore, research with time-series and experimental methods is encouraged to further support the hypotheses that follow from this analysis.

\section{Conclusions}

In this paper, we examined the associations among acculturation, HIV-related stigma, stress, and patient-HCP relationships among Asian American PLWHA. This is a vulnerable group with significant acculturation and psychosocial distress, yet there are limited reports on their experience navigating the U.S. healthcare systems and services. This exploratory study presents that acculturation is beneficial for patient-HCP relationships and that, therefore, it decreases PLWHA's perceived stigma and stress. Interventions designed to decrease HIV-related stress and stigma and support acculturation should be developed in order to enhance patient-HCP relationships. This will improve HIV care and reduce healthcare disparities in Asian Americans.

Acknowledgement We gratefully acknowledge all the study participants, without them, it is not possible to complete these projects. This publication resulted (in part) from research supported by NINR under Award Numbers [K23NR14107; PI: Chen, Wei-Ti], NIMHD [R03MD012210; PI: Chen, Wei-Ti] and NIMH [P30MH058107; PI: Shoptaw, Steven J.] \& [R25MH087217; PIs: Barbara Guthrie \& Trace Kershaw]. The contents of this article are solely the views of the authors and do not represent the official views of the National Institutes of Health.

\section{Compliance with ethical standards}

Conflict of interest The authors declare that they have no conflict of interest.

\section{References}

1. Pew Research Center. Key facts about Asian origin groups in the U.S. https://www.pewresearch.org/fact-tank/2019/05/22/ key-facts-about-asian-origin-groups-in-the-u-s/. Accessed 16 January 2020.

2. CDC. Diagnoses of HIV infection in the United States and dependent areas,2017. 2018. (HIV Surveillance Report ).

3. Chen WT, Guthrie B, Shiu CS, et al. Revising the American dream: how Asian immigrants adjust after an HIV diagnosis. J Adv Nurs. 2015;71(8):1914-25.

4. Whittal A, Rosenberg E. Effects of individual immigrant attitudes and host culture attitudes on doctor-immigrant patient relationships and communication in Canada. Int $\mathbf{J}$ Equity Health. 2015;14:108.

5. Chen WT, Shiu C, Yang JP, et al. A structural equation model of patient-healthcare provider relationships and HIVinfected patient outcomes in Chinese populations. AIDS Care. 2018;30(3):383-90.

6. Bakken S, Holzemer WL, Brown MA, et al. Relationships between perception of engagement with health care provider and demographic characteristics, health status, and adherence to therapeutic regimen in persons with HIV/AIDS. AIDS Patient Care STDS. 2000;14(4):189-97.

7. Chen WT, Starks H, Shiu CS, et al. Chinese HIV-positive patients and their healthcare providers: contrasting Confucian versus Western notions of secrecy and support. ANS Adv Nurs Sci. 2007;30(4):329-42.

8. Edmonds KA, Aspiras OG, Rose JP, et al. Cross-sectional evaluation of perceived health care provider engagement, self-efficacy, and ART adherence in people living with HIV/AIDS. AIDS Care. 2019;17:1-5.

9. Chen WT, Wantland D, Reid P, et al. Engagement with Health Care Providers Affects Self- Efficacy, Self-Esteem, Medication Adherence and Quality of Life in People Living with HIV. J AIDS Clin Res. 2013;4(11):256.

10. Gelaude DJ, Hart J, Carey JW, et al. HIV Provider Experiences Engaging and Retaining Patients in HIV Care and Treatment: "A Soft Place to Fall". J Assoc Nurses AIDS Care. 2017;28(4):491-503.

11. Tang K, Chen WT. HIV and religion in HIV-infected Asians and their families: A qualitative study. Appl Nurs Res. 2018;44:18-24.

12. Chen WT, Guthrie B, Shiu CS, et al. Acculturation and perceived stress in HIV+ immigrants: depression symptomatology in Asian and Pacific Islanders. AIDS Care. 2014;26(12):1581-5.

13. Chen WT, Barbour R. Life priorities in the HIV-positive Asians: a text-mining analysis in young vs old generation. AIDS Care. 2017;29(4):507-10.

14. Rajabiun S, Rumptz MH, Felizzola J, et al. The impact of acculturation on Latinos' perceived barriers to HIV primary care. Ethn Dis. 2008;18(4):403-8.

15. Glynn TR, Llabre MM, Lee JS, et al. Pathways to Health: an Examination of HIV-Related Stigma, Life Stressors, Depression, and Substance Use. Int J Behav Med. 2019;26(3):286-96. 
16. Levison JH, Bogart LM, Khan IF, et al. "Where It Falls Apart": Barriers to Retention in HIV Care in Latino Immigrants and Migrants. AIDS Patient Care STDS. 2017;31(9):394-405.

17. Sauceda JA, Wiebe JS, Chan K, Kutner BA, Simoni JM. Acculturation, family cohesion, and mental health among Latinos living with HIV on the US-Mexico border. Cultur Divers Ethnic Minor Psychol. 2018;24(3):453-8.

18. Berry JW, Kim U, Minde T, Mok D. Comparative studies of acculturative stress. International migration review. 1987;21(3):491-511.

19. Choi H. Cultural marginality: a concept analysis with implications for immigrant adolescents. Issues Compr Pediatr Nurs. 2001;24(3):193-206.

20. Bernal DR. Acculturation, acculturative stress, social status, and well-being among English language proficient immigrants.2014.

21. Suinn RM, Rickard-Figueroa K, Lew S, Vigil P. The Suinn-Lew Asian self-identity acculturation scale: An initial report. Educ Psychol Measur. 1987;47(2):401-7.

22. Berger BE, Ferrans CE, Lashley FR. Measuring stigma in people with HIV: psychometric assessment of the HIV stigma scale. Res Nurs Health. 2001;24(6):518-29.

23. Su X, Lau JT, Mak WW, et al. Development of the perceived stress scale for people living with HIV/AIDS in China. AIDS Patient Care STDS. 2008;22(12):989-98.

24. Yang YM, Wang HH. Acculturation and health-related quality of life among Vietnamese immigrant women in transnational marriages in Taiwan. J Transcult Nurs. 2011;22(4):405-13.

25. Kelcey B. A robust alternative estimator for small to moderate sample SEM: Bias-corrected factor score path analysis. Addict Behav. 2019;94:83-988.

26. Lt Hu, Bentler PM. Cutoff criteria for fit indexes in covariance structure analysis: Conventional criteria versus new alternatives. Struct Equ Modeling. 1999;6(1):1-55.
27. Tehranchi A, Neshat Doost HT, Amiri S, Power MJ. The Role of Character Strengths in Depression: A Structural Equation Model. Front Psychol. 2018;9:1609.

28. Villagran M, Hajek C, Zhao X, Peterson E, Wittenberg-Lyles E. Communication and culture: predictors of treatment adherence among Mexican immigrant patients. J Health Psychol. 2012;17(3):443-52.

29. Lazarus RS, Folkman S. Stress, appraisal, and coping. Springer publishing company. 1984.

30. Chen WT, Lee SY, Shiu CS, et al. Fatigue and sleep disturbance in HIV-positive women: a qualitative and biomedical approach. $\mathrm{J}$ Clin Nurs. 2013;22(9-10):1262-9.

31. Chen M, Liao Y, Liu J, et al. Comparison of Sexual Knowledge, Attitude, and Behavior between Female Chinese College Students from Urban Areas and Rural Areas: A Hidden Challenge for HIV/ AIDS Control in China. Biomed Res Int. 2016;2016:8175921.

32. Beyeza-Kashesya J, Wanyenze RK, Goggin K, et al. Stigma gets in my way: Factors affecting client-provider communication regarding childbearing among people living with HIV in Uganda. PLoS ONE. 2018;13(2):e0192902.

33. UNAIDS. 90-90-90: an ambitious treatment target to help end the AIDS epidemic. Geneva: Unaids. Accessed 01January 2017.

Publisher's Note Springer Nature remains neutral with regard to jurisdictional claims in published maps and institutional affiliations. 